\title{
Aortic valve sparing root surgery for Marfan syndrome
}

\author{
George Matalanis, Nisal K. Perera \\ Department of Cardiac Surgery, The Austin Hospital, Heidelberg, Australia \\ Correspondence to: Prof. George Matalanis. Department of Cardiothoracic Surgery, The Austin Hospital, PO BOX 555, Heidelberg, Victoria 3084, \\ Australia. Email: george.matalanis@austin.org.au.
}

\begin{abstract}
Aortic valve sparing root surgery (AVSRS) is a safe and durable alternative for patients with dilated roots or pure aortic regurgitation (AR), which avoids the risks of anticoagulation or valvular degeneration with prosthetic valves. Notwithstanding the theoretical challenges of greater tissue fragility in Marfan syndrome (MFS), AVSRS has been demonstrated to have equal outcomes in this condition as it does in those without MFS. The benefits of retaining the native aortic valve in this generally younger age group extend beyond those of avoiding the inconvenience and complications of prolonged exposure to anticoagulants and include ease of management for future aortic, cardiac and non-cardiac procedures which are the norm for these patients. The essential principles of AVSRS in MFS do not differ from those for the rest of the population. Successful repair and durable valve function depend on a sound understanding of the close interaction between the structure and function of this exquisitely designed piece of engineering. We are fortunate to have numerous tools in our surgical armamentarium to preserve these valves. It is the purpose of this paper to demystify the complex structure-function interactions of the aortic valve, thereby gaining an intuition for AVSRS. We will also elaborate on specific technical details of established techniques that we have found successful in preserving the normal function of these valves in the long term.
\end{abstract}

Keywords: Aortic root; Marfan

Submitted Oct 13, 2017. Accepted for publication Nov 01, 2017.

doi: $10.21037 /$ acs.2017.11.05

View this article at: http://dx.doi.org/10.21037/acs.2017.11.05

\section{Introduction}

The basic functional unit is the single cusp (Figure 1). It consists of leaflet tissue suspended like a sail from its fibrous attachment to the aortic wall and left ventricular outflow tract (LVOT).

It is useful to consider the impact of different dimensional variations on the shape of the leaflet. For practical purposes the basal leaflet attachment and geometric leaflet height are relatively fixed, so with regard to MFS and surgical manuipulation, the main changes in length we need to focus on are those of the leaflet free margin (FM) and the inter-commissural distance (ICD). Both elongation of the FM and reduction in ICD result in a sagging leaflet with a shallower cusp, while reduction of the FM and greater separation of the ICD result in a taller but narrower cusp (Figure 2).

The effect of variations in length is further complicated when the leaflets interact in three dimensions inside the cylinder of the aorta (Figure $3 A$ ), where the principal goal is to achieve a coaptation zone (CZ) to seal the valve in diastole (Figure 3B).

Nonetheless, one can ascertain the effect of aortic root dilatation by focusing on two planes: the sinotubular junction (STJ) and the annulus (AN), a circle intersecting the nadir of the leaflets. AN alteration is the easiest to understand, as it simply impacts on how closely the leaflets appose each other, without significantly altering their basic shape. AN dilatation results in a reduction in the $\mathrm{CZ}$ which, when severe enough leads to central aortic regurgitation (AR) (Figure 4).

STJ dilatation is more complex and affects not only leaflet interaction but also leaflet shape. At the level of the STJ circle, each leaflet's FM runs from one commissure to the adjacent one, contacting its neighbors along the radii of the circle (Figure $5 A$ ). In normal circumstances, the 3 


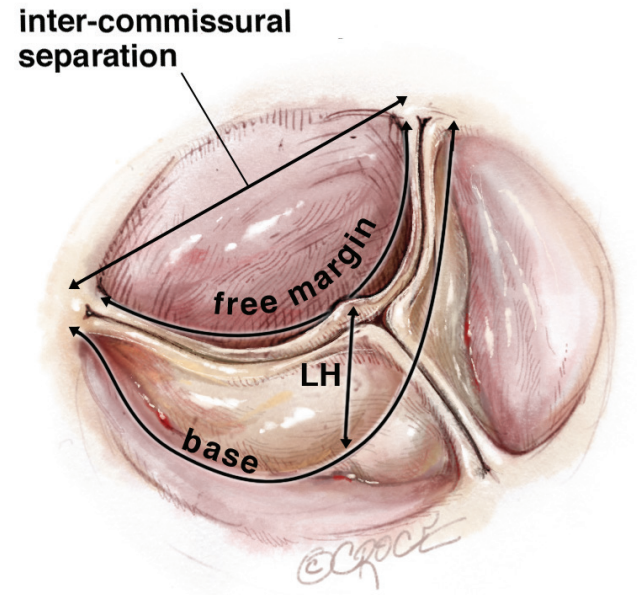

Figure 1 Single aortic cusp. LH, leaflet height. leaflets approximate the STJ in the open position. A simple application of the relationship of the perimeter of a circle to its diameter, suggests that the FM should nearly equal the STJ diameter. In the closed position, they run from one commissure to the next, touching each other at the center like radii of a circle. This again requires the FM to be nearly equal the STJ diameter. Thus the trileaflet design is ideal for opening and closing without excess redundancy or lack of co-aptation in the middle. With STJ dilatation, the diameter of the circle enlarges and hence the FM takes a straighter path from commissure to commissure, unable to contact its neighbors in the center (Figure 5B). In addition, the leaflet shape changes with STJ dilatation described previously (Figure 2), resulting in loss of the $\mathrm{CZ}$ with a different appearance compared to annular dilatation (Figure 6).
A

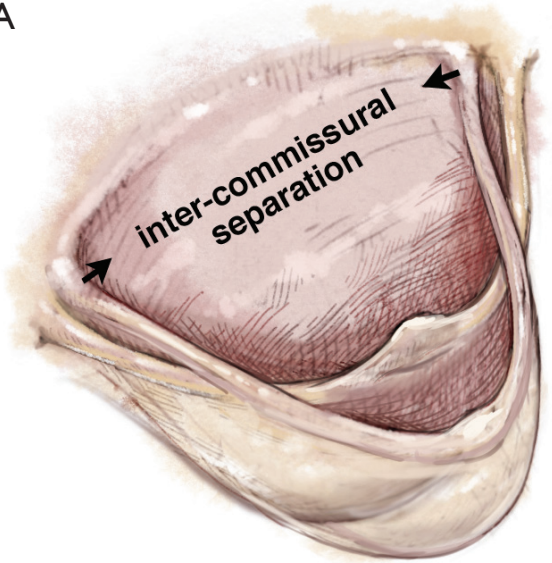

C

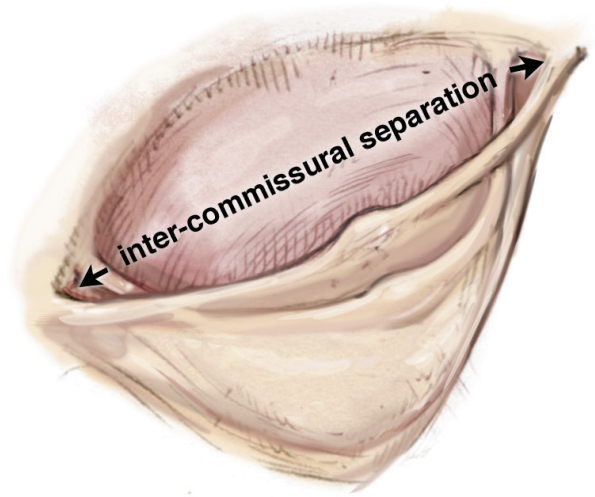

B

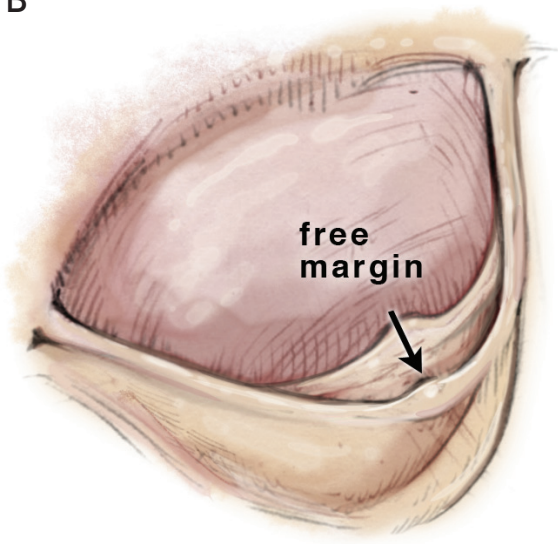

D

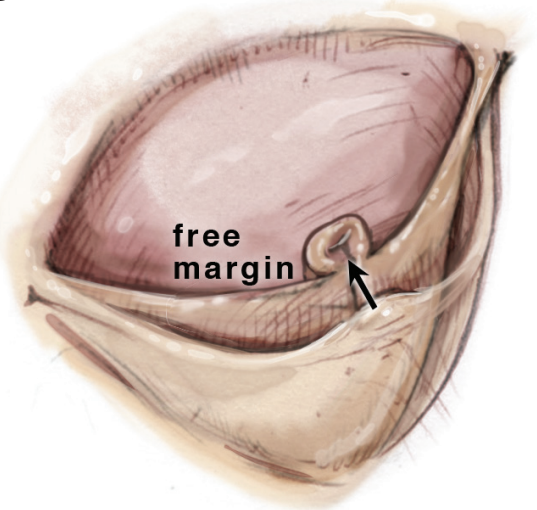

Figure 2 Consequences of changes in free margin. (A) Reduction in ICD; (B) elongation of FM; (C) elongation of ICD; (D) reduction in FM. ICD, inter-commissural distance; FM, free margin. 

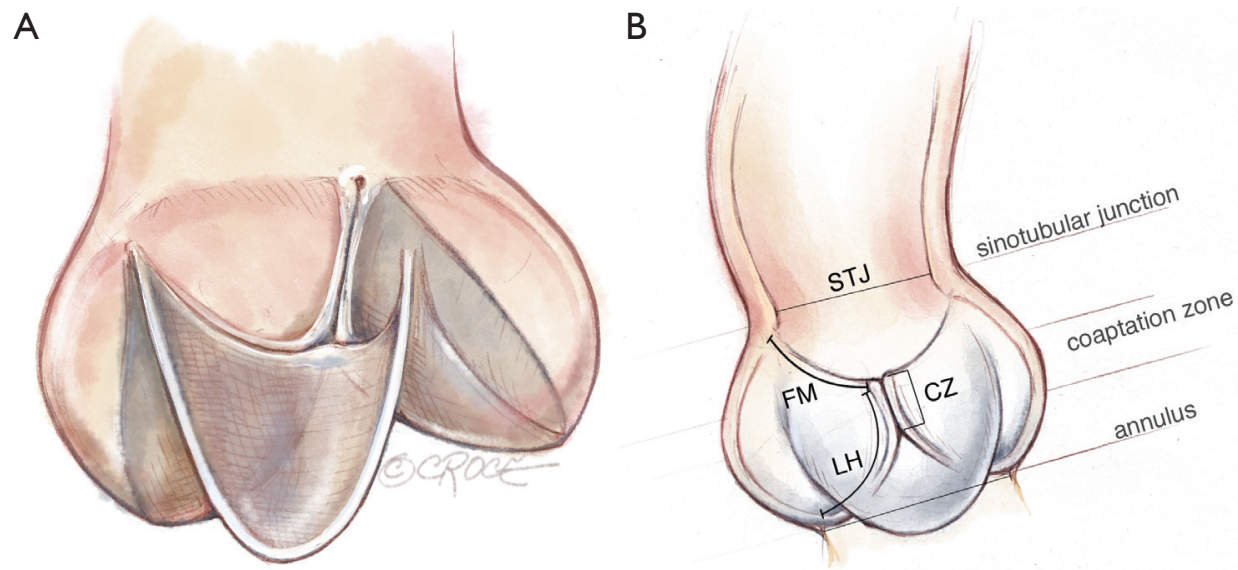

Figure 3 Leaflet interactions in the 3 dimensional aortic root. (A) Surface; (B) cross-sectional views. STJ, sinotubular junction; FM, free margin; CZ, coaptation Zone; LH, leaflet height; AN, annulus.
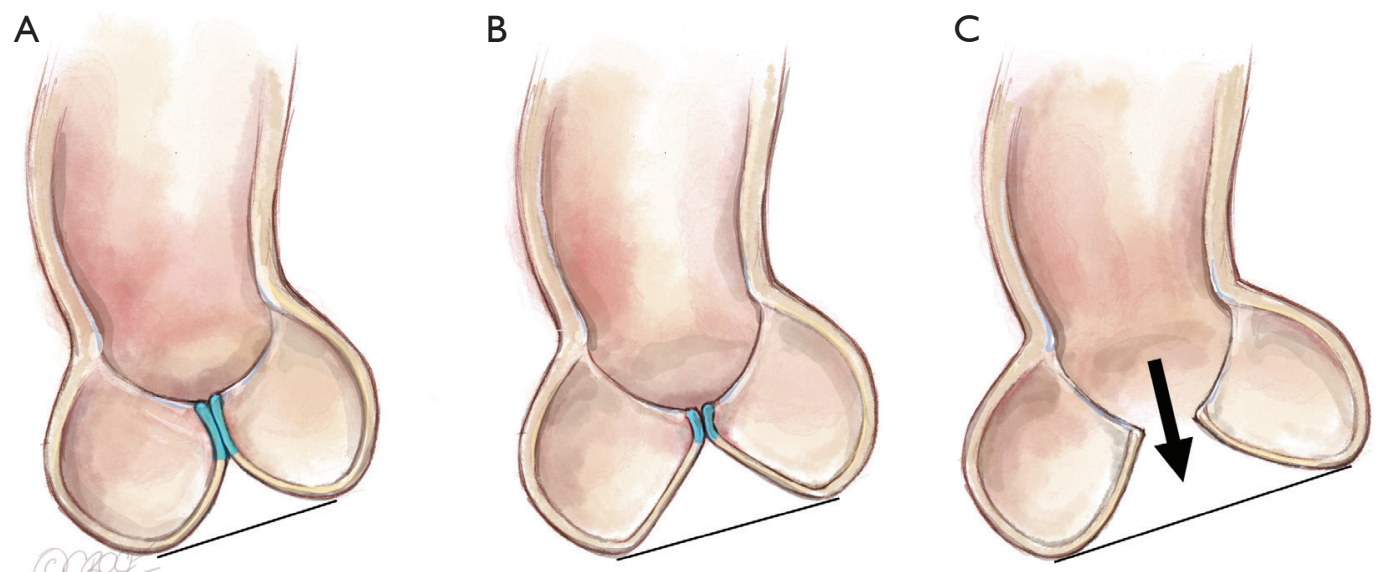

Figure 4 Impact of annular dilatation on the length of the coaptation zone (blue fill). (A) Normal valve; (B) moderate annular dilation, but still competent; (C) severe annular dilation, with central regurgitation.

A
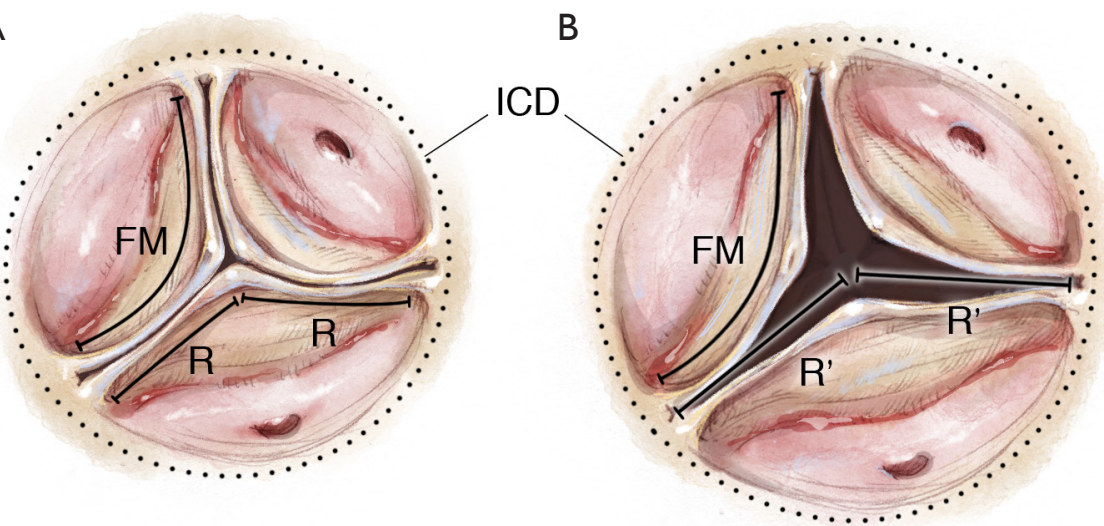

Figure 5 Interaction between STJ diameter, ICD and FM. (A) Normal valve; (B) STJ dilation. ICD, inter-commissural distance; FM, free margin; STJ, sinotubular junction; R, normal radius; R', radius of dilated STJ. 
A

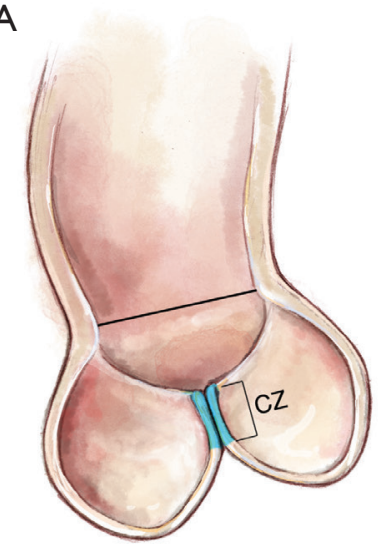

B

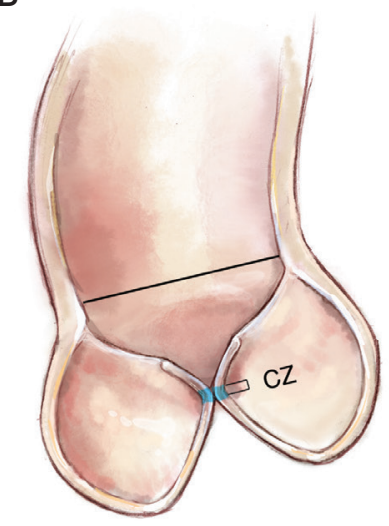

C

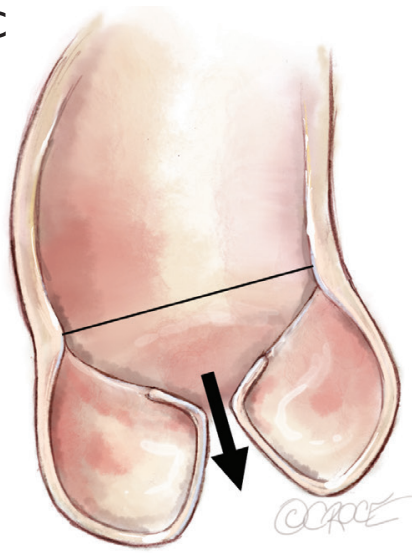

Figure 6 Leaflet alterations with STJ dilation. (A) Normal; (B) moderate STJ dilation; (C) severe STJ dilation. STJ, sinotubular junction; $\mathrm{CZ}$, coaptation zone.

A

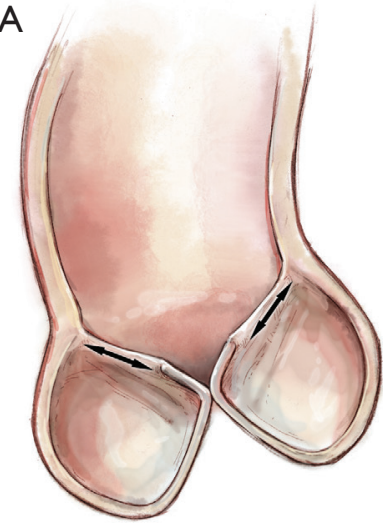

B

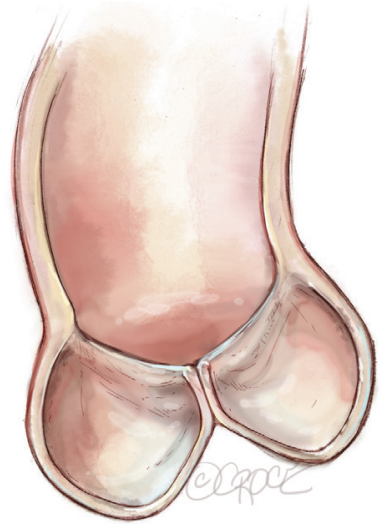

Figure 7 Stretching and elongation of the FM with STJ dilation. (A) High loading on FM; (B) compensation with elongated FM. FM, free margin; STJ, sinotubular junction.

Since the process of STJ dilatation is generally very gradual, compensatory stretching and elongation of the FM commonly occurs, preserving aortic competence for some time (Figure 7). This fact needs to be born in mind when correcting the STJ size in chronic root aneurysms, as this will induce symmetric leaflet prolapse.

The effect of isolated leaflet prolapse due to elongation of the FM on valvular competence is easily seen in Figure 8. In this scenario eccentric AR occurs with the jet arising centrally but directed away from the saggy leaflet. For example, a right coronary leaflet prolapse results in a posteriorly directed jet towards the anterior mitral valve leaflet.

The effect of symmetric prolapse is more subtle (Figure 9). It may seem as though the situation in Figure $9 B$ is preferable to normal, since the leaflet travelling horizontally would use less of its geometric length to reach its partners in the center, thus creating a longer CZ. However, loss of the 'prayer' angle results in two mechanical disadvantages. An angle with the horizontal, allows a component of the diastolic pressure to force the $\mathrm{CZ}$ more tightly together, thus increasing the friction of the seal, much like a ladder leaning against a wall. Secondly, with each leaflet leaning against its partners at the CZ, the FM is unloaded during diastole, thereby avoiding stress-related lengthening of the FM. Thus, it is easy to understand how prolapse begets further prolapse by losing the diastolic unloading of the FM.

Clearly when the leaflets sag below the plane of the AN (Figure 9C), diastolic pressure acting on the leaflets angled 

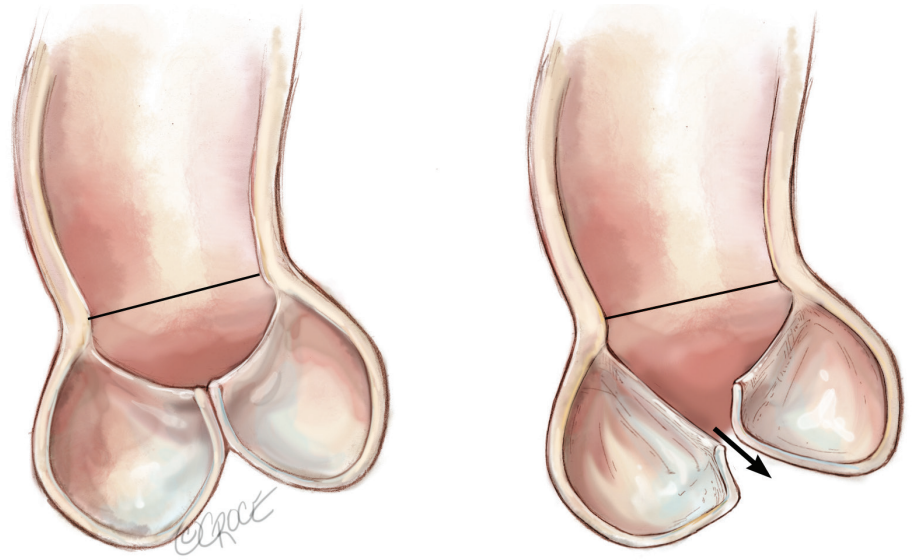

Figure 8 Isolated leaflet prolapse with eccentric AR. AR, aortic regurgitation.
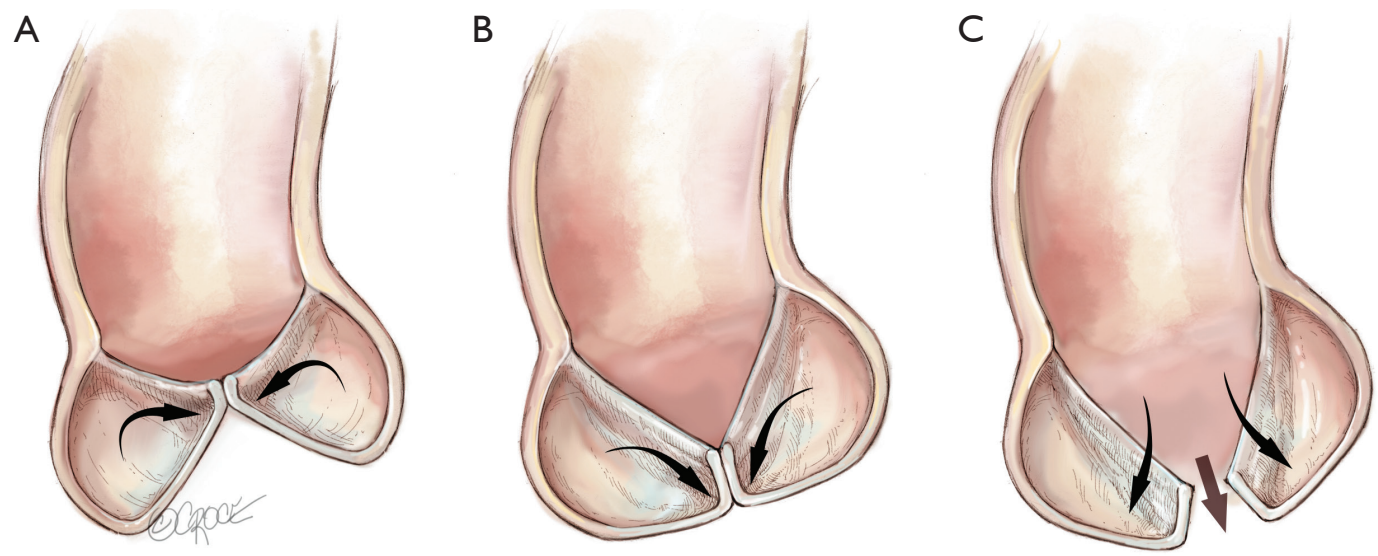

Figure 9 Effects of symmetric leaflet prolapse. (A) Normal; (B) mild; (C) severe.

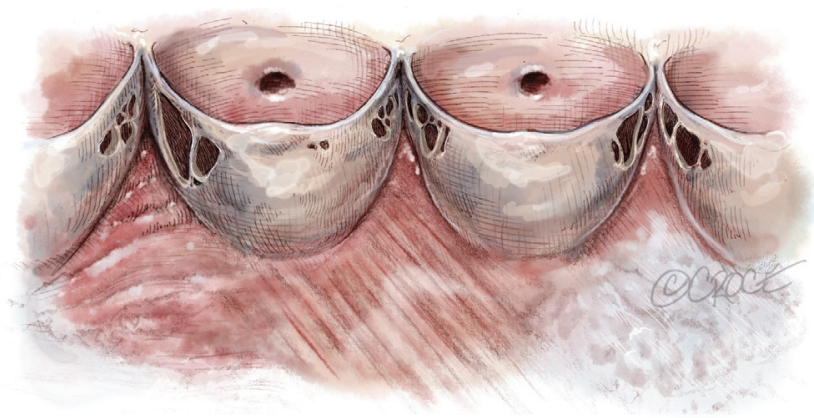

Figure 10 Aortic leaflet fenestrations.

below the horizontal will push them apart, resulting in severe AR.

As we are discussing AVSRS in the context of MFS, we will not concern ourselves with other pathologies, except for fenestrations which are quite common in these patients (Figure 10). Fortunately, most fenestrations lie in the $\mathrm{CZ}$ and do not contribute to aortic valve competence. The primary concern is to ensure that the FM is unloaded, as explained previously, to prevent future rupture.

If fenestrations are larger than normal and extend on to the belly of the leaflet outside the CZ, AR will result. This typically occurs in the commissural region, such that the AR jet is not only eccentric but also arises away from the center of coaptation. Another mechanism of AR is the sudden rupture of the thinned out portion of the FM attaching to the commissure. This effect is equivalent to severe elongation of the FM with severe prolapse.

\section{Operative techniques}

The foregoing discussion forms the basis of a logical 


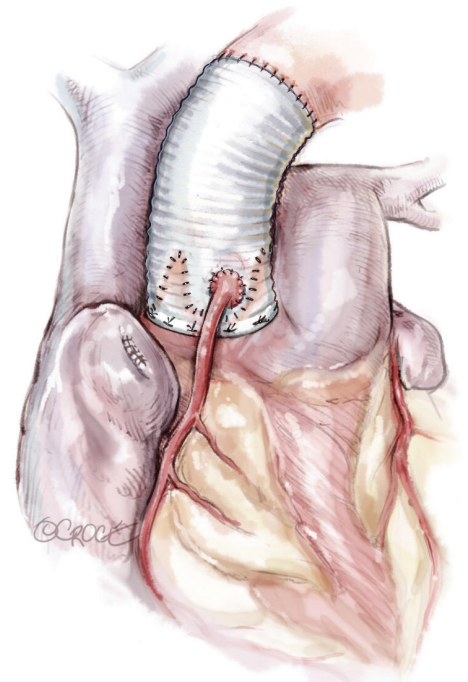

Figure 11 Re-implantation (David) technique.

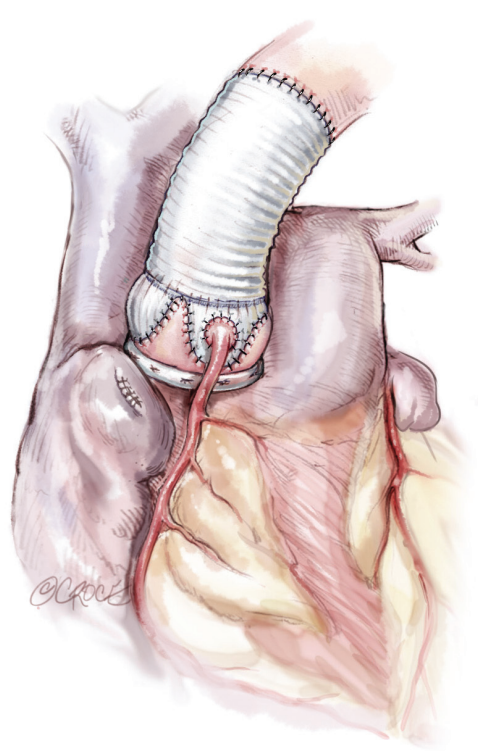

Figure 12 Remodelling with annuloplasty (Lansac) technique.

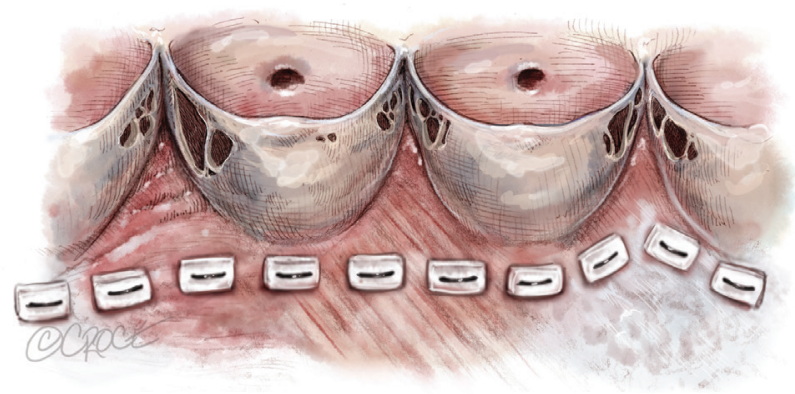

Figure 13 Line of suture implantation. approach to AVSRS. Both echocardiographic and intraoperative assessment should systematically interrogate the aortic root and valve, particularly with respect to the STJ and AN diameters, leaflet prolapse, as well as the quantity and direction of AR. In MFS, we are generally faced with dilatation of the diameters of the STJ, AN and all three sinuses.

In patients with longstanding root aneurysms, it is very likely that the adaptation to STJ dilatation has resulted in elongation of the leaflets' FM. AR, if present, tends to be central. Occasionally, we may see isolated leaflet prolapse secondary to a ruptured fenestration, resulting in AR which is centrally arising but eccentrically directed. Very large fenestrations may encroach on the belly of the leaflet, resulting in eccentric $\mathrm{AR}$ which arises near the perimeter of the valve in the region of a commissure.

Given the considerations above, the surgical plan must then be targeted to correct all pathologies. Correcting STJ and AN diameters, as well as removing the sinuses, generally requires a re-implantation (David) technique (Figure 11). Even if the AN is not significantly dilated, AN stabilization is required for MFS as omission of this has been shown to lead to poor long term outcomes $(1,2)$. Thus, if a remodeling technique (Yacoub) is preferred, then some form of annuloplasty should be added (Lansac) (3) (Figure 12).

The techniques of remodeling and re-implantation have been well described before $(4,5)$. We will avoid discussion regarding the controversies of the preferable type of Dacron prosthesis: simple tube, preformed sinuses or manually tailored. While there are theoretical advantages and disadvantages, clinical outcomes have not clearly supported one form over another. Nonetheless, certain details which may be glossed over in the routine operative description, have in our experience provided critical understanding to successful repair and are worth emphasizing.

\section{Annular sutures}

The aim is to mobilize the aortic root from its surrounding structures so as to exteriorize the LVOT from the cardiac skeleton. This then allows the annular sutures to be placed in a single plane which lies at the nadir of all three leaflets, except in the region of the membranous septum to avoid injury to the conductive tissue (Figure 13).

Thus, when the sutures are tied to the annuloplasty material, we achieve a symmetrical re-approximation of the 3 leaflets towards each other and a greater CZ. However, 


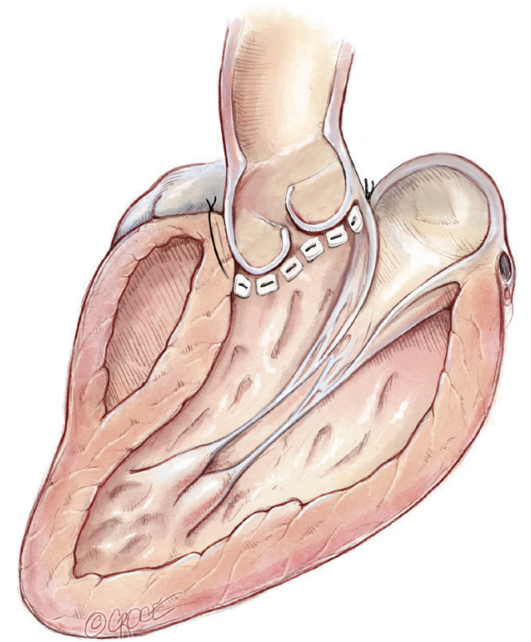

Figure 14 Sutures travelling obliquely upwards to exit clear of the RVOT can induce an asymmetric annuloplasty with poor coaptation.

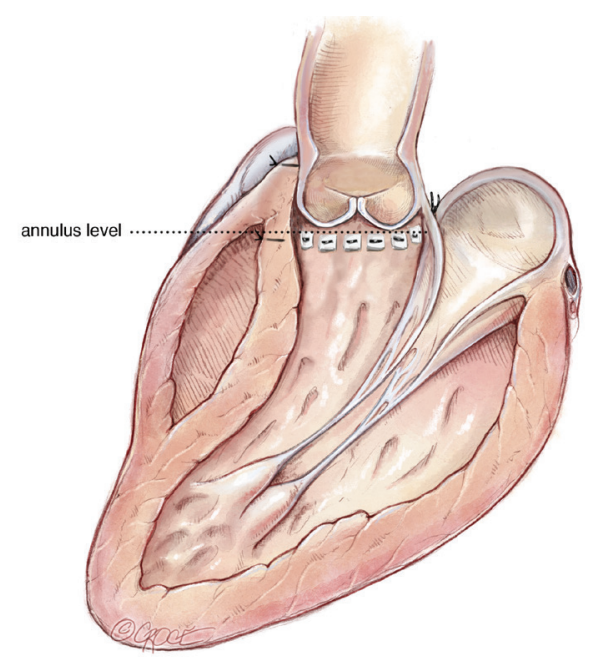

Figure 15 Deliberate detachment of some of the fibers of RV insertion can result in entering the RV cavity. Once the external graft is seated, the free wall of the RV can easily and securely reattached to the Dacron.

the external extent of mobilization of the aortic root does not always parallel this desirable internal line. This is particularly so in the region of the right ventricular outflow tract (RVOT), which may insert into the right sinus at a higher level (6).

If sutures in this region are inserted at the correct level internally but travel obliquely upwards to exit clear of the RVOT, we can induce an asymmetric annuloplasty, where the right leaflet fails to be exteriorized from the root and lies on a much lower plane than the other two leaflets. This may result in even worse coaptation than before (Figure 14).

In most cases, with minimal RV overlap, the needle can be passed horizontally to exit at the same external level after passing through the substance of the RV. In this way, we can still achieve symmetric annuloplasty. With more extensive RV overlap, it is necessary to deliberately detach some of the fibers of RV insertion. On occasion, this will result in entry into the RV cavity. However, that is not a major inconvenience, as once the external graft is seated, the free wall of the RV can be easily and securely reattached to the Dacron (Figure 15).

\section{Sizing of the graft}

There are numerous schemes for choosing the appropriate graft size $(7,8)$. The aim is to achieve an optimal CZ, without inducing an outflow gradient. In our experience, the method advocated by de Kerchove et al. (9) is logical and has been shown to yield predictable results. The height of the commissure between the non-coronary cusp and left coronary cusp, above the annular plane correlates well with the average geometric leaflet length, which in turn is $70 \%$ of the normal annular diameter (10).

Choosing the graft diameter to be equal to this length builds a $15 \%$ safety margin in achieving an optimal CZ. In fact, the annuloplasty effect is even greater for two reasons. Firstly, since the graft sits on the exterior surface of the LVOT, the effective internal diameter is even less by an average of $5 \mathrm{~mm}$ (11). Secondly, tying the horizontal mattress sutures (even with Teflon pledgets) will produce a purse string effect to some degree, adding to annular reduction. One needs to be mindful that despite the additional safety in downsizing the AN, excessive reduction can impair leaflet opening. It can also lead to a significant gradient across the valve.

\section{Induction of symmetric prolapse}

If the degree of STJ reduction is excessive for the given leaflet FM, then symmetric prolapse may be induced. This situation is not uncommon for a number of reasons. Unmodified Dacron tubes have the same diameter at the STJ as at the AN. Thus, while the size may be chosen to yield a large CZ, it may prove excessive for the STJ. Secondly, the FM of chronically dilated roots have often gradually lengthened under the extra strain, thus even the correct 

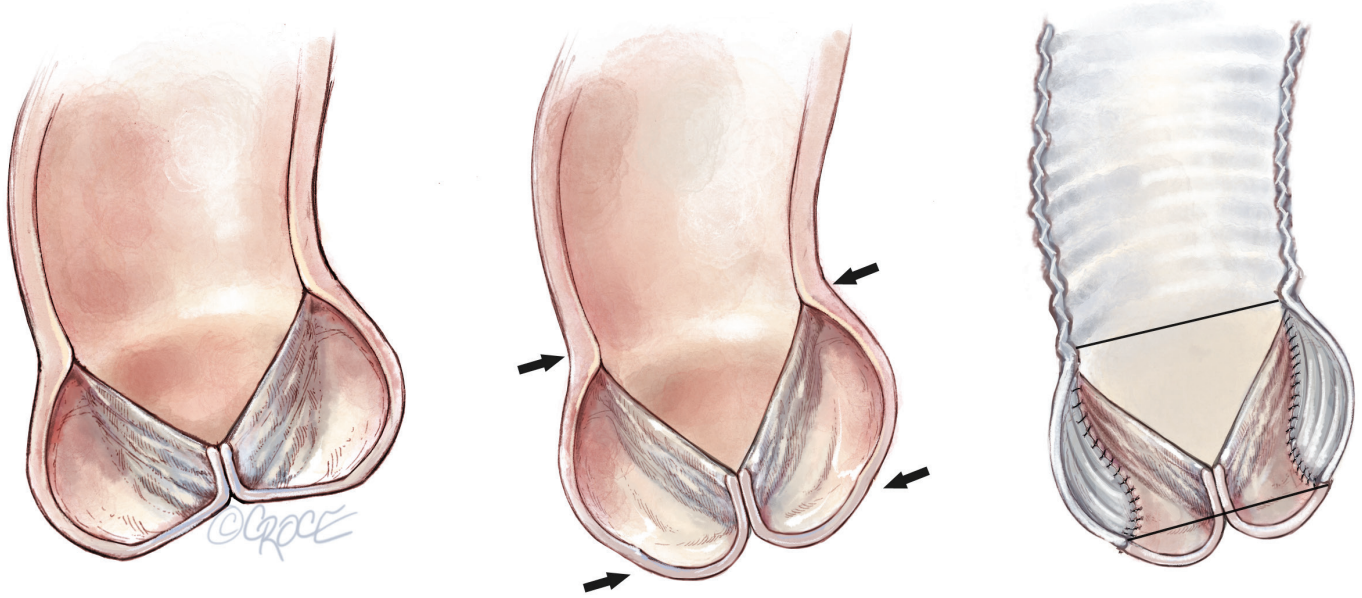

Figure 16 Symmetric leaflet prolapse after STJ diameter correction due to a lengthened FM. STJ, sinotubular junction; FM, free margin.

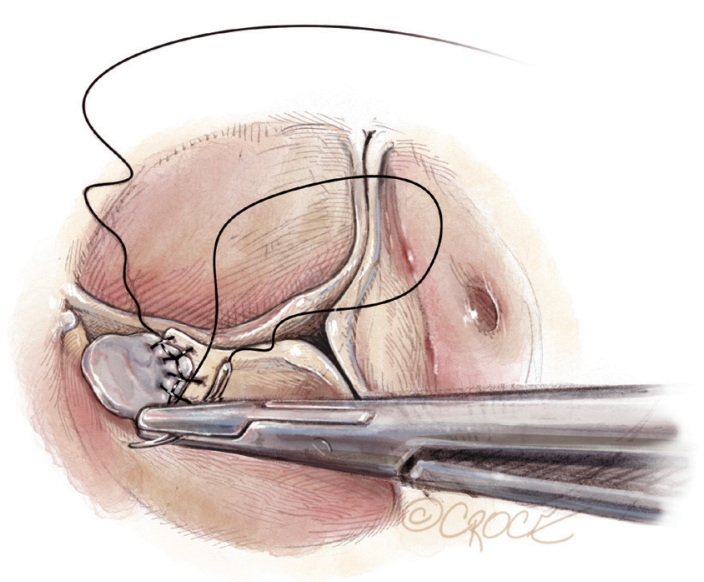

Figure 17 Repair of leaflet fenestration with autologous pericardial patch using a locking suture.

STJ diameter may become too small (Figure 16). Symmetric prolapse portends a poor medium term outcome $(12,13)$.

While this is easy to see in the post-bypass transoesophageal echocardiogram (TOE) by a coaptation level close to or below the $\mathrm{AN}$, it is more difficult to detect by direct visualisation at the completion of the repair. Fortunately, a specially designed caliper, the Schafers caliper (14), allows quantitative assessment of leaflet sagging. It has been shown that an effective measured height of $9 \mathrm{~mm}$ or more is desirable for optimal long term outcomes (15).

\section{Correction of leaflet prolapse}

Although there are several techniques for FM shortening, the most durable in our experience is that of central plication (16). In single leaflet prolapse, the common center of the two normal leaflets is used as the reference point to gauge the extent of central plication of the elongated leaflet. Unless the extent of plication required is minimal, it is important to extend the plication back into the redundant part of the belly of the leaflet to avoid billowing. The latter may appear to the untrained eye as prolapse on the postbypass TOE. The distinction comes from the fact that the CZ lies well above the annular level even though the belly of the leaflets may billow below. The long term effect of this is unknown, but it would seem best to avoid on the basis of sound engineering.

\section{Leaflet fenestrations}

As previously mentioned, most fenestrations don't require treatment unless they are particularly so large as to extend onto the belly beyond the $\mathrm{CZ}$ or if associated with a flail leaflet from rupture of the flimsy chordae. In such circumstances, we prefer the use of a small disc of untreated autologous pericardium, slightly larger than the size of the defect. We secure the patch with a 'locking' continuous 7-0 Prolene suture, to avoid any traction distortion on the remaining leaflet (Figure 17). It is also vital to delay any correction of FM elongation until this step is completed as a certain degree of FM shortening can occur despite the locking suture.

\section{Comments}

We have had experience with 193 AVSRS, of which 11\% 


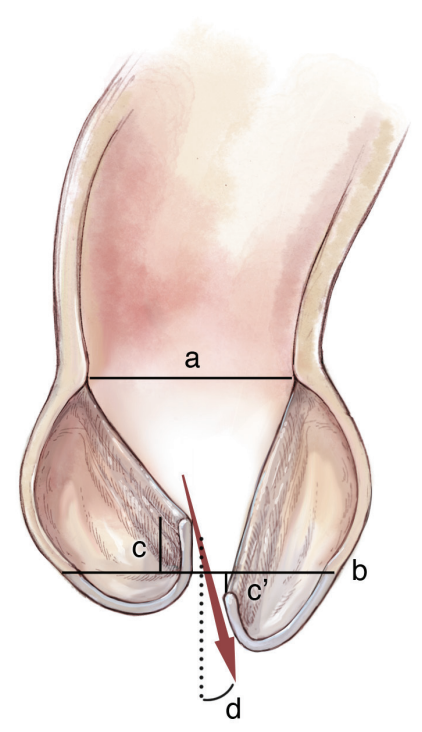

Figure 18 Schematic representation of the TOE measurements. (a) Sinotubular junction; (b) annulus; (c) annulus to tip of cusps; (d) angle of eccentric jet. TOE, trans-oesophageal echocardiogram.

were in MFS. Early outcomes were excellent with no mortality in MFS cases. Five-year freedom from significant AR was $95 \%$ in addition to $95 \%$ freedom from repeat AVR in the MFS group. There were no differences between MFS and non-MFS cohorts. Long term studies $(17,18)$ have also demonstrated excellent outcomes to 20 years with no influence of MFS provided that an annular stabilization procedure was included.

In our experience, the following factors have been found to be very important in achieving optimal results:

(I) Case selection

These are relative considerations and the threshold for abandoning repair will depend to some extent on the level of surgical experience.

(i) Avoid more than mild leaflet degeneration. This includes leaflets which are excessively stretched and thinned from grossly aneurysmal roots and presence of multiple large fenestrations;

(ii) Avoid patients with severe left ventricular dysfunction or multiple procedures, where less than perfect myocardial protection combined with longer duration of cross clamp may lead to poor cardiac performance post-operatively.

(II) Careful perioperative and intraoperative assessment of valvular and root anatomy and pathophysiology. This includes assessment on both pre-bypass TOE and direct operative observation of the following (Figure 18):

(i) Sinotubular junction;

(ii) Annulus;

(iii) Distance from annulus to tip of cusps;

(iv) Angle of eccentric jets.

(III) Post repair static testing:

After completion of the root repair and reimplantation of the coronary buttons, a very useful test is to clamp the new root and instill antegrade cardioplegia. The left ventricular vent is closed and the valve is examined with the TOE. Unsatisfactory findings include visualization of obvious aortic insufficiency and rapid left ventricular distension

(IV) Heeding the criteria for successful repair on postbypass TOE (19):

(i) No eccentric and no more than trace to mild central AR;

(ii) Symmetric leaflet coaptation, with tip of coaptation $9 \mathrm{~mm}$ or higher from the annular base;

(iii) A CZ length of more than $4 \mathrm{~mm}$;

(iv) Free leaflet opening with a mean gradient of no more than $10 \mathrm{mmHg}$.

Figure 18 illustrates an example of poor repair with (i) asymmetric leaflet coaptation; (ii) leaflet tip below annular plane; and (iii) a large eccentric jet.

(V) Liberal use of further periods of cross clamp to make minor adjustments.

Despite all the aforementioned care, leaflet FM correction may be slightly excessive or insufficient in one or more leaflets. A further short period of cross clamp to make small adjustments, can make a significant difference in achieving optimal long-term results compared to an early conversion to valve replacement or medium-term failure.

\section{Acknowledgements}

None.

\section{Footnote}

Conflicts of Interest: The authors have no conflicts of interest to declare.

\section{References}

1. de Oliveira NC, David TE, Ivanov J, et al. Results of surgery for aortic root aneurysm in patients with Marfan 
syndrome. J Thorac Cardiovasc Surg 2003;125:789-96.

2. Hanke T, Charitos EI, Stierle U, et al. Factors associated with the development of aortic valve regurgitation over time after two different techniques of valve-sparing aortic root surgery. J Thorac Cardiovasc Surg 2009;137:314-9.

3. Lansac E, Di Centa I, Vojacek J, et al. Valve sparing root replacement: the remodeling technique with external ring annuloplasty. Ann Cardiothorac Surg 2013;2:117-23.

4. David TE, Feindel CM, Webb GD, et al. Aortic valve preservation in patients with aortic root aneurysm: results of the reimplantation technique. Ann Thorac Surg 2007;83:S732-5; discussion S785-90.

5. Yacoub MH, Gehle P, Chandrasekaran V, et al. Late results of a valve-preserving operation in patients with aneurysms of the ascending aorta and root. J Thorac Cardiovasc Surg 1998 May;115:1080-90.

6. de Kerchove L, Jashari R, Boodhwani M, et al. Surgical anatomy of the aortic root: implication for valve-sparing reimplantation and aortic valve annuloplasty. J Thorac Cardiovasc Surg 2015;149:425-33.

7. Doty DB, Arcidi JM Jr. Methods for graft size selection in aortic valve-sparing operations. Ann Thorac Surg 2000;69:648-50.

8. Boodhwani M, de Kerchove L, El Khoury G. Aortic root replacement using the reimplantation technique: tips and tricks. Interact Cardiovasc Thorac Surg 2009;8:584-6.

9. de Kerchove L, Boodhwani M, Glineur D, et al. A new simple and objective method for graft sizing in valvesparing root replacement using the reimplantation technique. Ann Thorac Surg 2011;92:749-51.

10. Swanson M, Clark RE. Dimensions and geometric relationships of the human aortic valve as a function of

Cite this article as: Matalanis G, Perera NK. Aortic valve sparing root surgery for Marfan syndrome. Ann Cardiothorac Surg 2017;6(6):682-691. doi: 10.21037/acs.2017.11.05 pressure. Circ Res 1974;35:871-82.

11. Khelil N, Sleilaty G, Palladino M, et al. Surgical anatomy of the aortic annulus: landmarks for external annuloplasty in aortic valve repair. Ann Thorac Surg 2015;99:1220-6.

12. Pethig $\mathrm{K}$, Milz A, Hagl C, et al. Aortic valve reimplantation in ascending aortic aneurysm: risk factors for early valve failure. Ann Thorac Surg 2002;73:29-33.

13. Schafers HJ, Aicher D, Langer F, et al. Preservation of the bicuspid aortic valve. Ann Thorac Surg 2007;83:S740-5; discussion S785-90.

14. Schafers HJ, Bierbach B, Aicher D. A new approach to the assessment of aortic cusp geometry. J Thorac Cardiovasc Surg 2006;132:436-8.

15. Kunihara T, Aicher D, Rodionycheva S, et al. Preoperative aortic root geometry and postoperative cusp configuration primarily determine long-term outcome after valvepreserving aortic root repair. J Thorac Cardiovasc Surg 2012;143:1389-95.

16. Boodhwani M, de Kerchove L, Glineur D, et al. A simple method for the quantification and correction of aortic cusp prolapse by means of free margin plication. J Thorac Cardiovasc Surg 2010;139:1075-7.

17. Birks EJ, Webb C, Child A, et al. Early and long-term results of a valve-sparing operation for Marfan syndrome. Circulation 1999;100:II29-35.

18. David TE. Aortic valve sparing operations: outcomes at 20 years. Ann Cardiothorac Surg 2013;2:24-9.

19. le Polain de Waroux JB, Pouleur AC, Robert A, et al. Mechanisms of recurrent aortic regurgitation after aortic valve repair: predictive value of intraoperative transesophageal echocardiography. JACC Cardiovasc Imaging 2009;2:931-9. 